\title{
miR-27a-3p promotes the malignant phenotypes of osteosarcoma by targeting ten-eleven translocation 1
}

\author{
JIN LIU* ${ }^{*}$ MINGPENG LI* ${ }^{*}$ XIANCHENG LIU, FAN LIU and JIANWEI ZHU \\ Department of Orthopedics, Affiliated Hospital of Nantong University, Nantong, Jiangsu 226001, P.R. China
}

Received October 5, 2017; Accepted February 9, 2018

DOI: $10.3892 /$ ijo.2018.4275

\begin{abstract}
Osteosarcoma has become one of the most common primary malignant tumors affecting children and adolescents. Although increasing evidence has indicated that microRNAs (miRNAs or miRs) play important roles in the development of osteosarcoma, the expression of miR-27a-3p and its effects on osteosarcoma are not yet fully understood. In the present study, our data demonstrated that the expression of miR-27a-3p in osteosarcoma cell lines was significantly higher than that in the normal human osteoblastic cell line, hFOB 1.19 cell $(\mathrm{P}<0.01)$. In order to explore the role of miR-27a-3p in the development and progression of osteosarcoma, the expression of miR-27a-3p was inhibited by transfection of the MG-63 cells with miR-27a-3p inhibitor. The results revealed that the cell proliferative ability significantly decreased $(\mathrm{P}<0.01)$, the number of apoptotic cells significantly increased $(\mathrm{P}<0.01)$ and the number of cells passing through the Transwell membrane was significantly reduced in the group transfected with the miR-27a-3p inhibitor $(\mathrm{P}<0.01)$. At the same time, the expression of E-cadherin and $\alpha$-catenin was significantly upregulated $(\mathrm{P}<0.01)$, while the expression of vimentin was significantly downregulated in the group transfected with the miR-27a-3p inhibitor $(\mathrm{P}<0.01)$. Our results also revealed that the mRNA expression of ten-eleven translocation 1 (TET1) in the osteosarcoma cells was significantly downregulated compared with that in the hFOB 1.19 cells $(\mathrm{P}<0.01)$. Luciferase reporter system analysis indicated that $\mathrm{miR}-27 \mathrm{a}-3 \mathrm{p}$ recognized the TET1 3'-UTR. The protein expression of TET1 significantly increased in the group transfected with the miR-27a-3p inhibitor. The results from CCK- 8 assay, flow cytometric assay and Transwell invasion analysis revealed that TET1 knockdown inhibited the biological effects induced by the downregula-
\end{abstract}

Correspondence to: Professor Jianwei Zhu, Department of Orthopedics, Affiliated Hospital of Nantong University, 20 Xishi Road, Nantong, Jiangsu 226001, P.R. China

E-mail: zhujianwei_nt@163.com

*Contributed equally

Key words: microRNA-27a-3p, osteosarcoma, ten-eleven translocation 1, apoptosis, epithelial-mesenchymal transition, invasion tion of miR-27a-3p. Taken together, the findings of this study indicate that miR-27a-3p is upregulated, while TET1 is downregulated in human osteosarcoma cells. miR-27a-3p inhibition suppresses the proliferation and invasion of osteosarcoma cells, and promotes cell apoptosis via the negative regulation of TET1. miR-27a-3p/TET1 may thus be a potential target for the treatment of osteosarcoma.

\section{Introduction}

Osteosarcoma is the most common primary malignant bone tumor, which originates from mesenchymal tissue. It is characterized by spindle-shaped stromal cells that produce bone-like tissue $(1,2)$. It usually occurs in adolescents with metaphysis, mainly around the knee joint (3). According to statistical data, the incidence of osteosarcoma is ranked first among all primary malignant bone tumors (4). The malignant degree of osteosarcoma is extremely high. The prognosis is poor, and pulmonary metastasis can occur within several months, with the survival rate being only approximately $60 \%$ following amputation (5-7).

At present, radical surgery is still the first choice of treatment for osteosarcoma, and is accompanied by adjuvant chemotherapy at high doses (8-10). With the promotion of radical surgery and high-dose chemotherapy, the 5-year survival rate of patients with osteosarcoma has increased to approximately $70 \%$ (11). However, there a considerable number of patients with this disease still suffer from tumor recurrence and distant metastasis following complex treatment (12). Distant metastasis is one of the most important reasons for the failure of treatment in the majority of patients with osteosarcoma (13-15). The main reason for the distant metastasis of osteosarcoma is the abnormal expression of tumor metastasisrelated genes and metastasis suppressor genes $(16,17)$.

In recent years, some progress has been made in the etiology, development, diagnosis and treatment of osteosarcoma; however, this progress has been very gradual, and the specific pathogenesis of the disease is not yet clear $(17,18)$. In addition, the lack of an effective target for the diagnosis and treatment of osteosarcoma has made prognosis and treatment difficult. Therefore, the identification of biological targets for osteosarcoma may have important theoretical and clinical implications (19).

MicroRNAs (miRNAs or miRs) are single-stranded noncoding RNAs of 21-23 nucleotides in length (20-22). miRNAs 
regulate gene expression via the specific binding to target genes (23). Over the past decades, a large number of miRNAs have been identified and are believed to play an important role in the occurrence, differentiation, proliferation and apoptosis of cells $(24,25)$. Recent studies have indicated that alterations in miRNA expression are associated with the occurrence, development, diagnosis and prognosis of cancers (26-28).

It has been previously demonstrated that miR-27 can regulate the expression of genes involved in cell metabolism, differentiation, apoptosis, migration and immunity (29,30); On the other hand, an abnormal expression of miR-27 has been found in a variety of tumors and diseases (31-33). miR-27 regulates cell apoptosis, cell cycle progression and cell proliferation, as well as processes via the post-transcriptional regulation of different molecules, which indicates that miR-27 is a key molecule in the development of tumors $(30,34)$. Pan et al demonsrated that miR-27a promoted osteosarcoma cell proliferation, migration and invasion (33). However, the molecular mechanisms underlying the role of miR-27a in the development and progression of osteosarcoma remain largely unexplored. In this study, revere transcription-quantitative PCR (RT-qPCR) was used to detect the expression of miR-27a-3p in human osteosarcoma cell lines, and Cell Counting kit- 8 (CCK-8) assay, flow cytometry and Transwell assay were used to examine the effects of miR-27a-3p on the proliferation, apoptosis and invasion of osteosarcoma cells.

It has been previously demonstrated that epigenetic modification plays a very important role in the occurrence and development of tumors. The degree of DNA methylation in prostate cancer and breast cancer is closely related to the progression of the tumor (35). The expression of ten-eleven translocation 1 (TET1) in prostate cancer and breast cancer tissues is downregulated, and it has been found that the deletion of TET1 promotes the invasion and growth of tumor cells, and promotes the metastasis of tumor cells in a xenograft tumor model; TET1 overexpression can reduce cell invasion and inhibit the formation of xenograft tumors $(36,37)$. Online software (http://www.targetscan.org) analysis was used to predict the possible miR-27a-3p target genes, whereby TET1 was identified as a miR-27a-3p target gene. In addition, the luciferase reporter gene system was used to determine whether TET1 is a regulatory target gene of miR-27a-3p. Western blot analysis was used to examine the effects of miR-27a-3p on the expression of TET1, and the mRNA expression of TET1 in osteosarcoma cell lines as detected by RT-qPCR. Further validation of the cell biological functions of miR-27a-3p in MG-63 cells through the regulation of TET1 was achieved by assessing the cell biological functions of miR-27a-3p in MG-63 cells through the regulation of TET1 via the overexpression of TET1.

Therefore, the findings of this study may provide the experimental basis for the application of miR-27a-3p and TET1 in the treatment of osteosarcoma.

\section{Materials and methods}

Cell lines and cell culture. Normal human osteoblasts (hFOB 1.19 cells), were cultured in mixed medium containing $0.5 \mathrm{mM}$ sodium pyruvate, $2.5 \mathrm{mM}$ L-glutamine (Gln), $15 \mathrm{mM}$ HEPES and $10 \%$ fetal bovine serum (FBS) (DMEM and
F12 medium were mixed 1:1). The human osteosarcoma MG-63 and MNNG/HOS Cl \#5 cells were cultured in EMEM containing $2.0 \mathrm{mM}$ L-glutamine (Gln), $1.0 \mathrm{mM}$ sodium pyruvate, $0.1 \mathrm{mM}$ non-essential amino acid, $1.5 \mathrm{~g} / \mathrm{ml}$ sodium bicarbonate and 10\% FBS Eagle's BSS. Saos-2 cells were cultured in McCoy's 5A medium with $1.5 \mathrm{~g} / \mathrm{ml}$ sodium bicarbonate, $1.5 \mathrm{mM}$ L-glutamine (Gln) 15\% FBS. The U-2OS cells were cultured in McCoy's 5A medium containing $1.5 \mathrm{~g} / \mathrm{ml}$ sodium bicarbonate, $1.5 \mathrm{mM}$ L-glutamine (Gln) and 10\% FBS.

All cell lines are purchased from the Cell Bank of the Chinese Academy of Sciences (Shanghai, China). FBS, sodium pyruvate, L-glutamine, non-essential amino acid and basic culture medium for cell culture were all purchased from Invitrogen (Carlsbad, CA, USA). The above-mentioned cells were cultured under conditions of $37^{\circ} \mathrm{C}, 5 \% \mathrm{CO}_{2}$ and saturated humidity. The growth state of the cells was observed under an inverted microscope (Leica DM IL LED; Leica, Wetzlar, Germany). When the cells reached a $70-80 \%$ fusion state, they were digested with $0.25 \%$ trypsin (Invitrogen). Cells at the logarithmic growth phase were collected for use in the experiments.

$R T-q P C R$. RT-qPCR was used to detect the expression of miR-27a-3p in normal human osteoblasts (hFOB 1.19) and osteosarcoma cell lines (MG-63, MNNG/HOS Cl \#5, Saos-2 and U-2OS). All cell lines were collected in vitro and the TaqMan miRNA isolation kit was used to extract the RNA. TaqMan microRNA Assay and TaqMan Universal PCR Master Mix (Applied Biosystems, Foster city, CA, USA) were used to detect the expression of mature miR-27a-3p, using U6 as an internal reference. The primers used were as follows: miR-27a-3p forward, 5'-ACA CTC CAG CTG GGT TCA CAG TGG CTA AG-3' and reverse, 5'-TGG TGT CGT GGA GTC G-3'; and U6 forward, 5'-CTC GCT TCG GCA GCA CA-3' and reverse, 5'-AAC GCT TCA CGA ATT TGC GT-3'. The thermocycling conditions used for PCR were as follows: Firstly pre-heating at $95^{\circ} \mathrm{C}$ for $10 \mathrm{~min}$, followed by 40 cycles of denaturation at $95^{\circ} \mathrm{C}$ for $10 \mathrm{sec}$, annealing at $60^{\circ} \mathrm{C}$ for $60 \mathrm{sec}$, and extension at $72^{\circ} \mathrm{C} 90 \mathrm{sec}$. All experiments were conducted in triplicate. The RT-qPCR experimental results were analyzed by using the relative quantification method (38).

Following transfection of the MG-63 cells with miR-27a-3p inhibitor and inhibitor negative control for $48 \mathrm{~h}$, the TaqMan miRNA isolation kit was used to respectively extract the RNA from the cells in each group, and miR-27a-3p expression in the MG-63 cells of each group was detected by RT-qPCR as described above.

The mRNA expression of TET1 in the osteosarcoma cells was also detected by RT-qPCR. Total RNA was extracted from the osteosarcoma cells using TRIzol reagent (Invitrogen), and the first strand cDNA was synthesized using the First Strand cDNA Synthesis kit (Qiagen, Hilden, Germany). GAPDH was used as an internal reference gene to detect the mRNA expression of mRNA TET1. The primers used were as follows: TET1 forward, 5'-GCCAGCAGAAGACCAACT-3' and reverse, 5'-TCCAGAGGCACAACAACA-3'; GAPDH forward, 5'-GACCTGACCTGCCGTCTA-3' and reverse, 5'-AGGAGTGGGTGTCGCTGT-3'.

The thermocycling conditions were as follows: Firstly pre-heating at $95^{\circ} \mathrm{C}$ for $10 \mathrm{~min}$, followed by 40 cycles of 
denaturation at $95^{\circ} \mathrm{C}$ for $10 \mathrm{sec}$, annealing at $60^{\circ} \mathrm{C}$ for $60 \mathrm{sec}$, and extension at $72^{\circ} \mathrm{C} 90 \mathrm{sec}$. All experiments were conducted in triplicate. The RT-qPCR experimental results are analyzed using the relative quantification method (38).

Cell treatment. Normal cultured MG-63 cells were inoculated into 6 -well plates at a density of $3 \times 10^{5}$ cells/well. After cell adherence, according to the instructions of the provider of Lipofectamine 2000 transfection reagent (Invitrogen), transfection with miR-27a-3p inhibitor, inhibitor negative control (provided by GenePharma, Shanghai, China), TET1 siRNA or siRNA control (scramble) (provided by Guangzhou RiboBio Co., Ltd., Guangzhou, China) was carried out. At the same time, the normal control group consisted of untransfected cells. MEM culture medium without serum was used to dilute the miR-27a-3p inhibitor, inhibitor negative control, TET1 siRNA and siRNA control, respectively. The diluted Lipofectamine 2000 was mixed with miR-27a-3p inhibitor or inhibitor negative control or TET1 siRNA or siRNA control. After gentle mixing, incubation was carried out at room temperature for $20 \mathrm{~min}$ to form the complex. The compound was added to the culture plate of MG63 cells, which was placed in an incubator at $37^{\circ} \mathrm{C}$ and a $5 \% \mathrm{CO}_{2}$ volume fraction. After $5 \mathrm{~h}$, the culture medium was replaced with MEM medium containing $10 \%$ fetal bovine serum and culture was continued for $48 \mathrm{~h}$.

CCK- 8 assay. The normal cultured MG-63 cells were inoculated into 96 -well plates with $1 \times 10^{6} / \mathrm{ml}$, and the volume of each well was $100 \mu \mathrm{l}$. Following cell adherence, and following the instructions of the provider of Lipofectamine 2000 (Invitrogen), transfection with miR-27a-3p inhibitor, or inhibitor negative control was carried out, and the normal control group consisted of untransfected cells. Following transfection for 24, 48 and $72 \mathrm{~h}, 10 \mu \mathrm{l}$ CCK-8 solution (Sigma-Aldrich, St. Louis, MO, USA) were added to each well of a 96-well plate and the plates were then placed in a $\mathrm{CO}_{2}$ incubator for continuous cultivation for $48 \mathrm{~h}$. A microplate reader (BioTek Instruments, Inc., Winooski, VT, USA) is used to detect the OD value at the $450-\mathrm{nm}$ position.

Analysis of cell apoptosis. The normal cultured MG-63 cells were inoculated into 96-well plates. Following transfection, the MG-63 cells were digested by trypsin, and washed with PBS. Annexin V-FITC and PI staining solution (Beyotime Institute of Biotechnology, Haimen, China) were then added. The 96-well plates were placed at room temperature for $15 \mathrm{~min}$. After screen filtering, flow cytometric analysis was performed (flow cytometer; BD Biosciences, Palo Alto, CA, USA) and FCM cellQuest software (BD Biosciences) was used for cell counting. FACsuite software (BD Biosciences) was adopted to analyze the data.

Transwell invasion assay. The cell invasive ability was examined by Transwell invasion assay. The MG- 63 cells transfected miR-27a-3p inhibitor and inhibitor negative control were added to the upper section of a Transwell chamber (Corning Inc., Corning, NY, USA). The bottom of the Transwell chamber was filled with $500 \mu \mathrm{l}$ of medium containing $10 \%$ FBS. Following $48 \mathrm{~h}$ of incubation, the MG-63 cells on the top surface of the chamber were removed using a cotton swab. The MG-63 cells which had passed through the Transwell chamber were fixed with $4 \%$ paraformaldehyde for $15 \mathrm{~min}$ at room temperature and stained with a crystal violet solution (Beyotime Institute of Biotechnology) for $10 \mathrm{~min}$. A total of 8 visual fields were observed at random using a Leica microscope (Leica Microsystems, Bensheim, Germany). Image pro plus 6.0 software (Media Cybernetics Inc., Silver Spring, MD, USA) was used to count the cells passing through the Transwell polycarbonate film. The cells passing through the Transwell polycarbonate film were the invading cells.

Luciferase reporter gene system assay. TargetScan (http:// www.targetscan.org/) was used to predict the gene targeted by miR-27a-3p, and TET1 was predicted as a potential target. The luciferase reporter plasmid was constructed, which included the miR-27a-3p complementary binding site wild-type 3'-UTR sequence segments of TET1 (TET13'-UTR WT) and mutations of 3'-UTR sequence segments of TET1 (TET1 3'-UTR MUT). The normal cultured 293 cells (the Cell Bank of the Chinese Academy of Sciences, Shanghai, China) was inoculated into the wells of a 6 -well plate at a density of $3 \times 105 / \mathrm{ml}$ cells/well, and the volume of each pore was 1,000 $\mu \mathrm{l}$. The luciferase plasmid containing TET1 3'-UTR WT or TET1 3'-UTR MUT and miR-27a-3p mimic or mimic negative-control (GenePharma) were co-transfected into the 293 cells. Following transfection for $48 \mathrm{~h}$, the 293 cells were collected and the luciferase activity was determined using a microplate reader (BioTek Instruments, Inc.).

Western blot analysis. Total protein was extracted from the osteosarcoma cells using RIPA buffer (Beyotime Institute of Biotechnology) and the quantitative analysis of the protein concentration was performed using a BCA assay kit (Beyotime Institute of Biotechnology). A total of $50 \mu \mathrm{g}$ of total protein was separated for each lane, and then was transferred onto PVDF membranes (Bio-Rad, Hercules, CA, USA). Subsequently, the PVDF membranes were blocked with blocking buffer containing 5\% skim milk powder, $10 \mathrm{mM}$ Tris- $\mathrm{HCl}, 150 \mathrm{mM}$

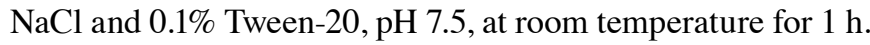
This was followed by the addition of the primary antibodies [mouse anti-human vimentin monoclonal antibody (1:2,000 dilution; ab8979), rabbit anti-human E-cadherin polyclonal antibody (1:1,000 dilution; ab15148), rabbit anti human TET1 (ab105475) and $\alpha$-catenin monoclonal (ab51032) antibodies (1:2,000 dilution) (all from Abcam, Cambridge, MA, USA) and rabbit anti-human $\beta$-actin monoclonal antibody $(1: 1,000$ dilution; A5441; Sigma-Aldrich)] and incubation of the PVDF membranes at $4^{\circ} \mathrm{C}$ overnight, followed by continuous incubation with secondary antibodies [HRP-labeled goat anti-rabbit IgG (A6154) or goat anti-mouse IgG (A4416); Sigma-Aldrich; $1: 2,000$ dilution] at $37^{\circ} \mathrm{C}$ for $1 \mathrm{~h}$. Finally, the PVDF membranes were treated with enhanced chemiluminescence reagent (Pierce, Rockford, IL, USA), and exposed to medical X-ray film. The relative content of vimentin, E-cadherin, $\alpha$-catenin and TET1 was determined using PDQuest software (Bio-Rad).

Statistical analysis. SPSS17.0 statistical analysis software was used for the statistical analysis of the experimental data. The t-test was used for comparisons between 2 groups. 


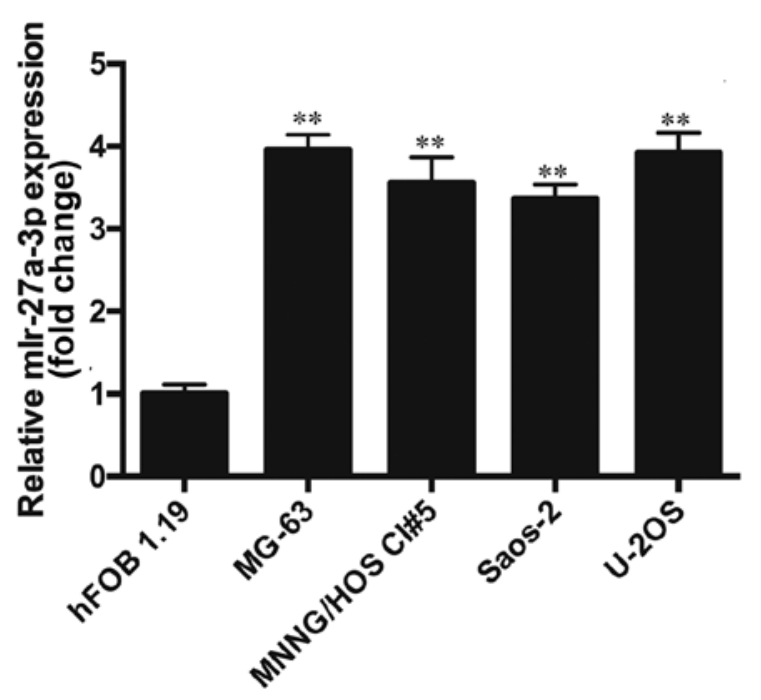

Figure 1. Relative expression of miR-27a-3p in osteosarcoma cell lines The expression of miR-27a-3p was analyzed by RT-qPCR in normal human osteoblasts cells (hFOB 1.19) or osteosarcoma cell lines (MNNG/HOS Cl \#5, MG-63, U-2OS and Saos-2). ${ }^{* *} \mathrm{P}<0.01$ vs. hFOB 1.19 cells. All experiments were run in triplicate. Data are expressed as the means \pm SEM.

One-way ANOVA followed by Tukey's post hoc test was used for comparisons of 3 or more groups (groups or variables) to determine statistical significance. The results were considered to be statistically significant at a value of $\mathrm{P}<0.05$.

\section{Results}

miR-27a-3p is upregulated in human osteosarcoma cells. The results from RT-qPCR revealed that the expression of miR-27a-3p in the osteosarcoma cell lines (MNNG/HOS $\mathrm{Cl}$ \#5, MG-63, U-2OS and Saos-2) was significantly higher than that in the normal human osteoblasts (hFOB 1.19) $(\mathrm{P}<0.01)$. The expression of $\mathrm{miR}-27 \mathrm{a}-3 \mathrm{p}$ in the osteosarcoma cell lines was approximately 3.5 -fold higher than that in the hFOB 1.19 cells (Fig. 1), which indicated that the upregulation of miR-27a-3p may play an important role in the development of osteosarcoma. As the expression of miR-27a-3p was highest in the MG-63 cells, these cells were selected for use in transfection experiments. In the future, we aim to perform further studies using multiple cell lines.

miR-27a-3p inhibition suppresses cell proliferation and induces cell apoptosis. In order to examine the effects of miR-27a-3p on osteosarcoma cells, miR-27a-3p inhibitor was transfected into the MG-63 cells, and the expression of miR-27a-3p was detected by RT-qPCR. The results revealed that the expression of miR-27a-3p was significantly lower in the group transfected with the miR-27a-3p inhibitor than in the normal control group (untransfected cells; $\mathrm{P}<0.01$ ), and the negative control group $(\mathrm{P}<0.01)$ (Fig. 2). These data indicated that the use of miR-27a-3p inhibitor effectively inhibited its expression.

The effect of miR-27a-3p on MG-63 cell viability was determined by CCK- 8 assay. The results revealed that the OD450 value was significantly lower in the group transfected with the miR-27a-3p inhibitor than in the normal control group

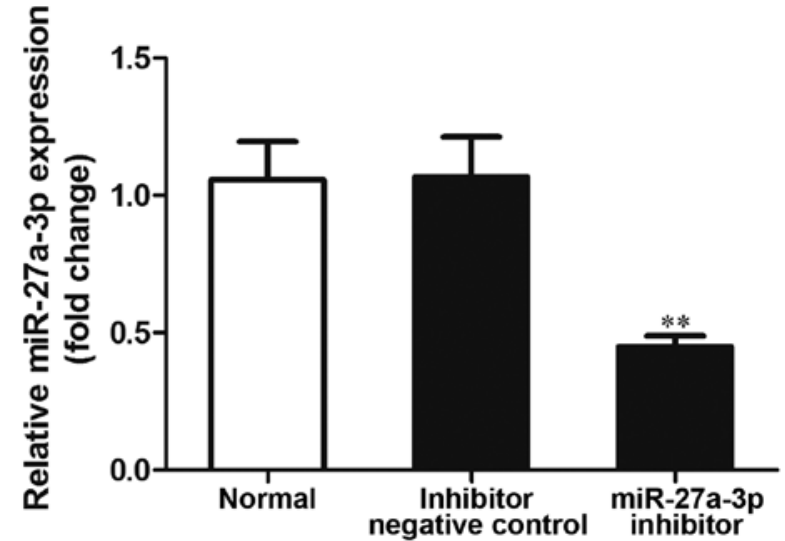

Figure 2. Expression of miR-27a-3p in transfected MG-63 cells. miR-27a-3p expression was determined in MG-63 cells transfected with miR-27a-3p inhibitor, inhibitor negative control and empty vector (Normal). ${ }^{* *} \mathrm{P}<0.01$ vs. Normal or inhibitor negative-control. All experiments were run in triplicate. Data are expressed as the means \pm EM.

and negative control group $(\mathrm{P}<0.01)$ (Fig. 3A), which demonstrated that the inhibition of miR-27a-3p expression reduced the ability of the MG-63 cells to proliferate, suggesting that miR-27a-3p promotes the proliferation of MG-63 cells.

The effect of miR-27a-3p on the apoptosis of the MG-63 cells was detected by flow cytometric analysis. The results revealed that the proportion of apoptotic cells in the group transfected with the miR-27a-3p inhibitor was significantly higher than that in the normal control group and negative control group $(\mathrm{P}<0.01)$ (Fig. 3B). These data demonstrated that the inhibition of miR-27a-3p promoted MG-63 cell apoptosis.

Downregulation of miR-27a-3p suppresses the invasive ability of the MG-63 cells. The effects of miR-27a-3p on the invasive ability of the MG-63 cells were examined by Transwell invasion assay. The results indicated that the number of cells that passed through the Transwell chamber was significantly reduced in the group transfected with the miR-27a-3p inhibitor compared with the normal control group and negative control group $(\mathrm{P}<0.01)$ (Fig. 4). These data demonstrated that the downregulation of miR-27a-3p suppressed the invasive ability of the osteosarcoma cells.

In order to explore the mechanisms responsible for the promoting effects of miR-27a-3p on the invasion of osteosarcoma cells, the expression levels of epithelial-mesenchymal transition (EMT)-associated proteins (vimentin, E-cadherin and $\alpha$-catenin) were detected by western blot analysis. The results revealed that, compared with the control group, the expression of vimentin was significantly decreased $(\mathrm{P}<0.01)$, while the expression of E-cadherin and $\alpha$-catenin was significantly increased $(\mathrm{P}<0.01)$ in the group transfected with the miR-27a-3p inhibitor (Fig. 5). These data indicated that the downregulation of miR-27a-3p suppressed the expression of vimentin and induced the expression of E-cadherin and $\alpha$-catenin, thus preventing EMT.

TET1 is the target gene of miR-27a-3p. The TargetScan tools (http://www.targetscan.org/) were used to predict the potential targets of miR-27a-3p. The results indicated that TET1 was one of the potential targets of miR-27a-3p. Subsequently, 

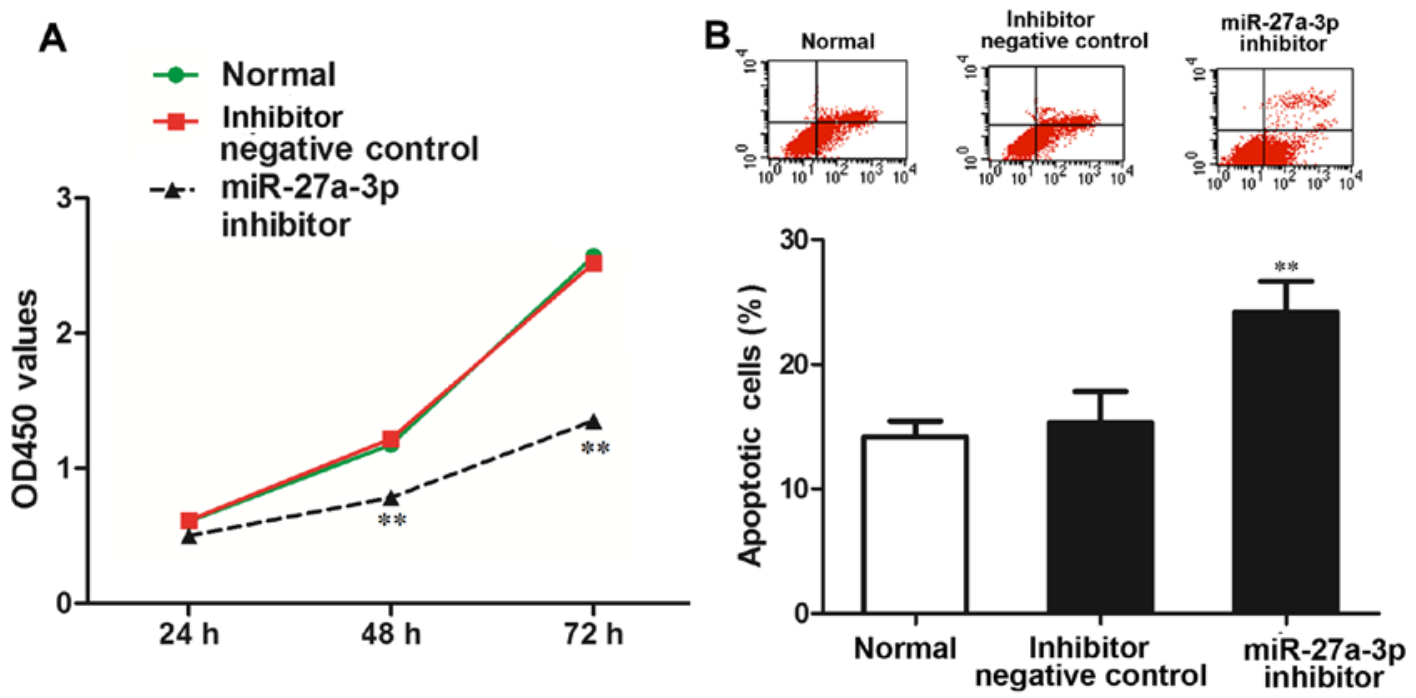

Figure 3. Effect of miR-27a-3p on cell viability and apoptosis. (A) Effect of miR-27a-3p on cell viability detected by CCK-8 assay; (B) effect of miR-27a-3p on cell apoptosis detected by flow cytometric analysis. ${ }^{* *} \mathrm{P}<0.01 \mathrm{vs}$. Normal or inhibitor negative control. All experiments were run in triplicate. Data are expressed as the means $\pm \mathrm{EM}$.
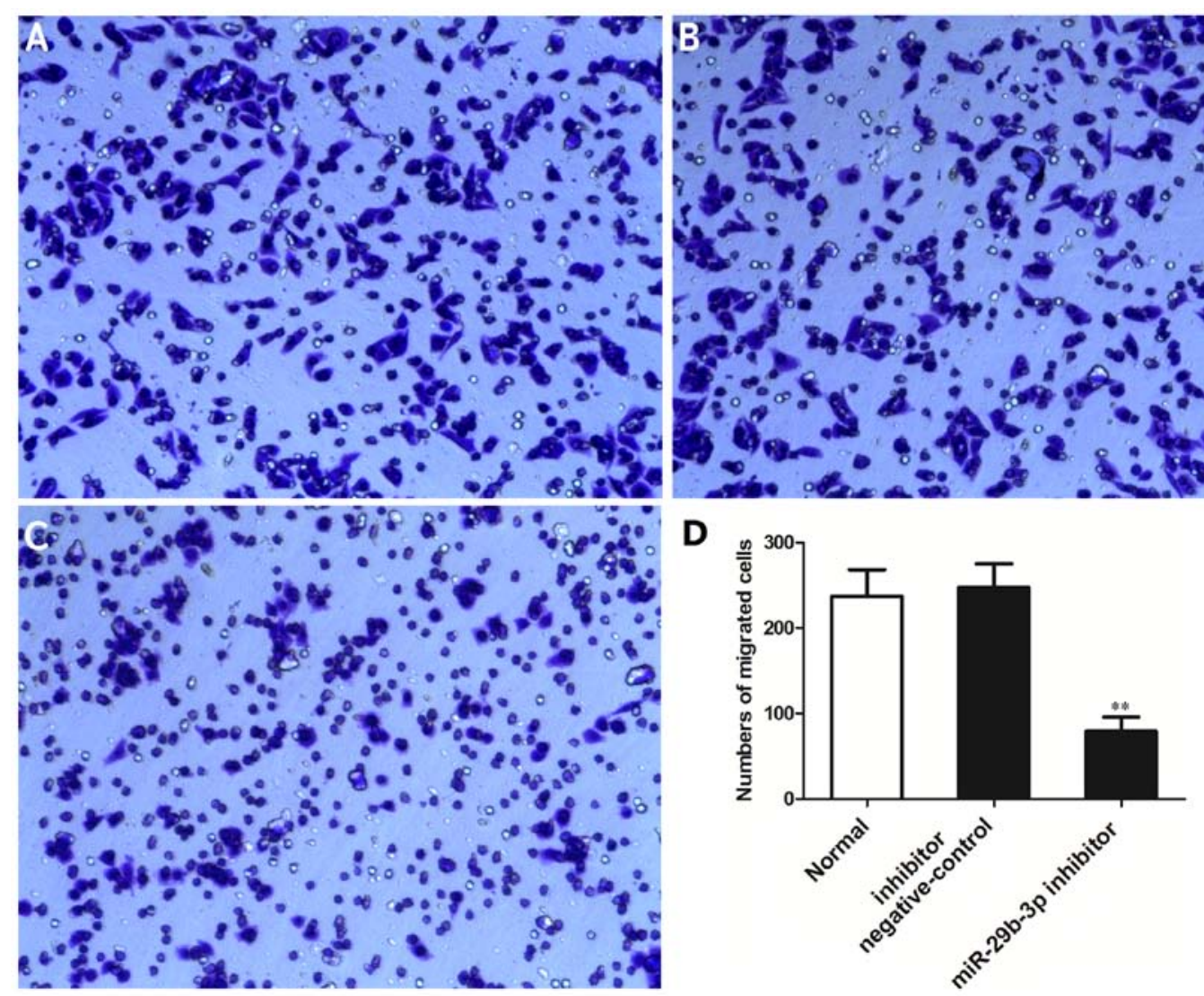

Figure 4. Effect of miR-27a-3p on cell invasion examined by Transwell assay. (A) Normal group; (B) miRNA inhibitor negative control transfection group; (C) miR-27a-3p inhibitor transfection group. (D) Quantitative analysis of the numbers of invading cells; ${ }^{* *} \mathrm{P}<0.01$ vs. Normal or inhibitor negative control. Data are expressed as the means $\pm \mathrm{EM}$.

the dual-luciferase reporter gene system was used to verify whether TET1 was the target of miR-27a-3p. The results revealed that the luciferase activity was significantly decreased in the 293 cells co-transfected with miR-27a-3p mimic and TET1 3'-UTR (WT), compared to the cells co-transfected with the negative control (mimic negative control) and
TET1 3'-UTR (WT) or miR-27a-3p mimic and TET1 3'-UTR (MUT) $(\mathrm{P}<0.01)$ (Fig. 6). These results indicated that miR-27a-3p may directly interact with the target site of the TET1 3'-UTR.

In order to explore the role of miR-27a-3p in regulating the expression of TET1, western blot analysis was used to detect 

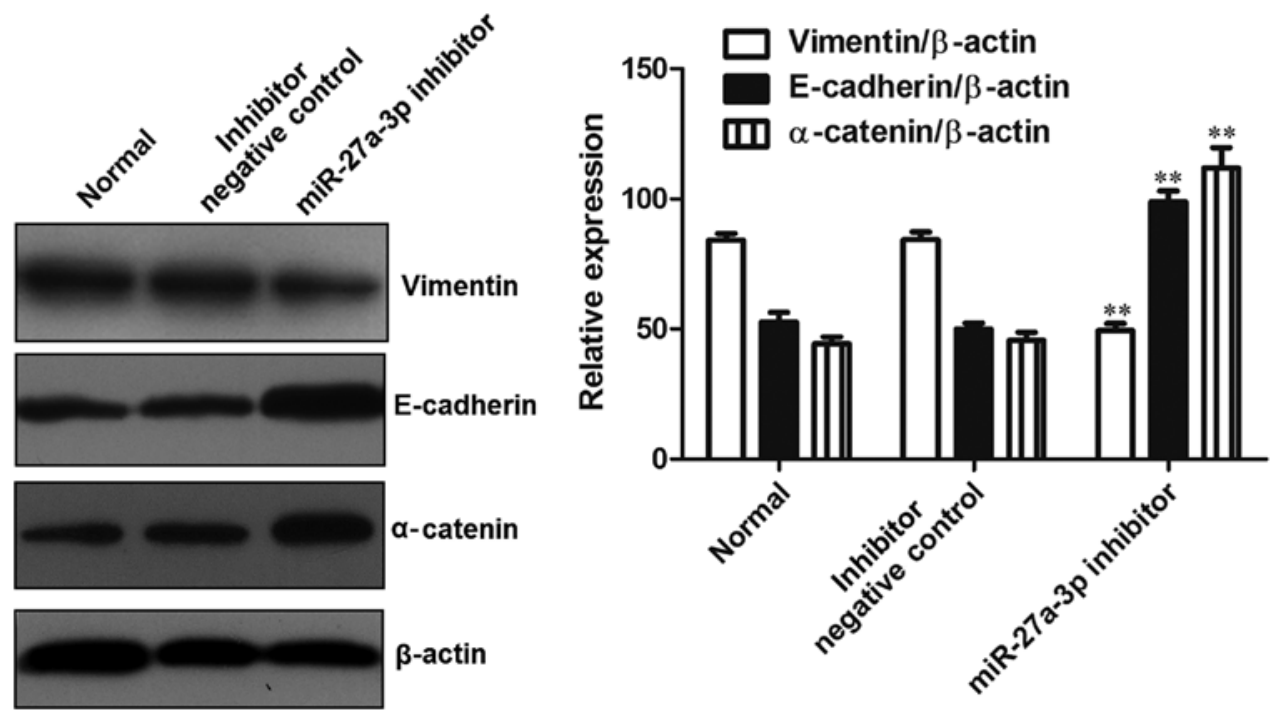

Figure 5. Effect of miR-27a-3p on the expression of EMT-related proteins in osteosarcoma cells detected by western blot analysis. ${ }^{* *} \mathrm{P}<0.01$ vs. Normal or inhibitor negative control. Data are expressed as the means \pm EM.
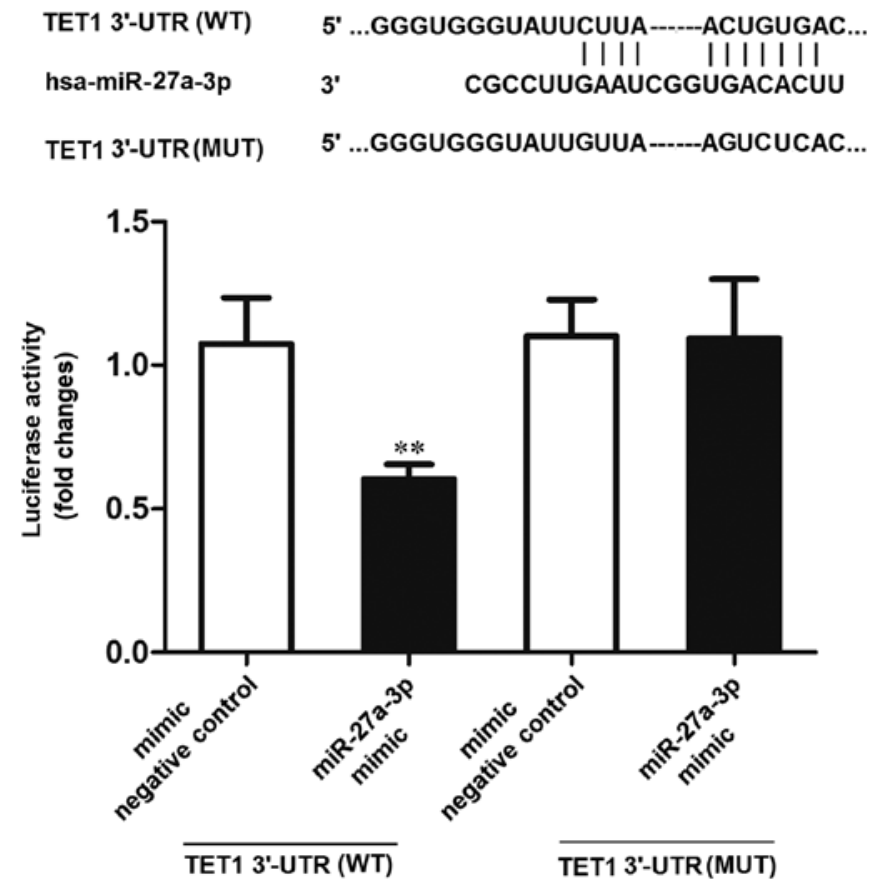

Figure 6 . TET1 is the target of miR-27a-3p validated by the luciferase reporter gene system. ${ }^{* *} \mathrm{P}<0.01$ vs. the mimic negative control group. Data are expressed as the means \pm EM.

the protein expression of TET1 in the MG-63 cells transfected with $\operatorname{miR}-27 \mathrm{a}-3 \mathrm{p}$. The results revealed that the protein expression of TET1 was significantly lower in the group transfected with the miR-27a-3p mimic $(\mathrm{P}<0.01)$ (Fig. 7A), which demonstrated that miR-27a-3p inhibited the protein expression of TET1 in the MG-63 cells.

Since TET1 was found to be a target gene of miR-27a-3p, and miR-27a-3p expression was upregulated in human osteosarcoma cells, we then wished to examine TET1 expression in osteosarcoma cells. RT-qPCR was used to detect the mRNA expression of TET1 in the human osteosarcoma cell lines. The results revealed that the mRNA expression of TET1 in the osteosarcoma cell lines (MNNG/HOS Cl \#5, MG-63, U-2OS and Saos-2) was significantly lower than that in the normal human osteoblasts (hFOB 1.19) $(\mathrm{P}<0.01)$. Its expression in the osteosarcoma cells was approximately $1 / 3$ lower than that in the hFOB1.19 cells (Fig. 7B). These data demonstrated that TET1 was the target gene of miR-27a-3p in osteosarcoma cells.

miR-27a-3p affects cellular biological functions by regulating TET1. Our data indicated that TET1 was a target gene of miR-27a-3p. In order to verify that miR-27a-3p plays a biological role in osteosarcoma cells by regulating TET1, miR-27a-3p inhibitor and TET1 siRNA were co-transfected into the MG-63 cells using Lipofectamine 2000. We further verified that the expression of TET1 was regulated by miR-27a-3p in the MG-63 cells. The results revealed that the mRNA expression of TET1 was significantly decreased $(\mathrm{P}<0.01)$ in the group transfected with TET1 siRNA, and was upregulated in the group co-transfected with miR-27a-3p inhibitor and TET1 siRNA compared to the group transfected with TET1 siRNA $(\mathrm{P}<0.01)$. The mRNA expression of TET1 did not differ significantly between the normal control group (Normal) and the siRNA negative control group (siRNA scramble) (Fig. 8A).

CCK-8 assay, flow cytometry and Transwell assay were used to further verify that miR-27a-3p plays a biological role by regulating the expression of TET1. The results from CCK-8 assay revealed that the OD450 value was significantly increased in the group transfected with TET1 siRNA compared to that in the group co-transfected with miR-27a-3p inhibitor and TET1 siRNA $(\mathrm{P}<0.01)$, which indicated that the viability of the MG-63 cells was enhanced in the group transfected with TET1 siRNA compared to that in the group co-transfected with miR-27a-3p inhibitor and TET1 siRNA. Thus, the inhibition of TET1 expression may promote the proliferation of MG-63 osteosarcoma cells. The OD450 value of the MG-63 cells in the group co-transfected with miR-27a-3p inhibitor and TET1 siRNA did not differ significantly compared with that in the normal control group and siRNA negative control group (siRNA scramble) (Fig. 8B). The results from flow cytometry 
A
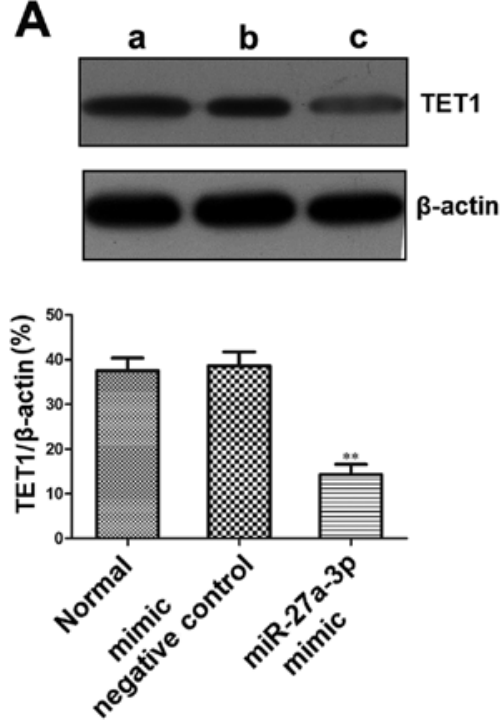

B

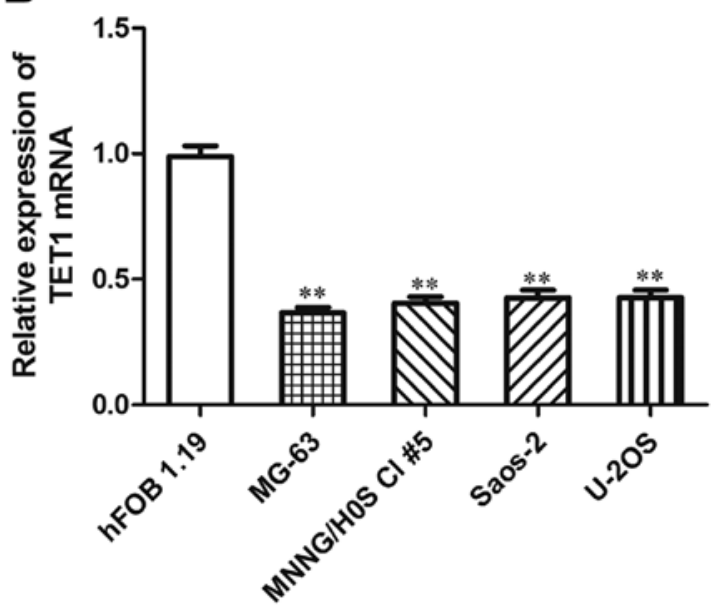

Figure 7. Effect of miR-27a-3p on the mRNA and protein expression of TET1 in osteosarcoma cells. (A) Effect of miR-27a-3p on the protein expression of TET1 detected by western blot analysis $\left({ }^{* * *} \mathrm{P}<0.01 \mathrm{vs}\right.$. mimic negative control and Normal group). Lane a, normal group; lane b, mimic negative control transfection group; and lane c, miR-27a-3p mimic transfection group. (B) The mRNA expression of TET1 in human osteosarcoma cell lines detected by RT-qPCR $\left({ }^{* *} \mathrm{P}<0.01\right.$ vs. hFOB 1.19 normal osteoblasts). Data are expressed as the means $\pm \mathrm{EM}$.
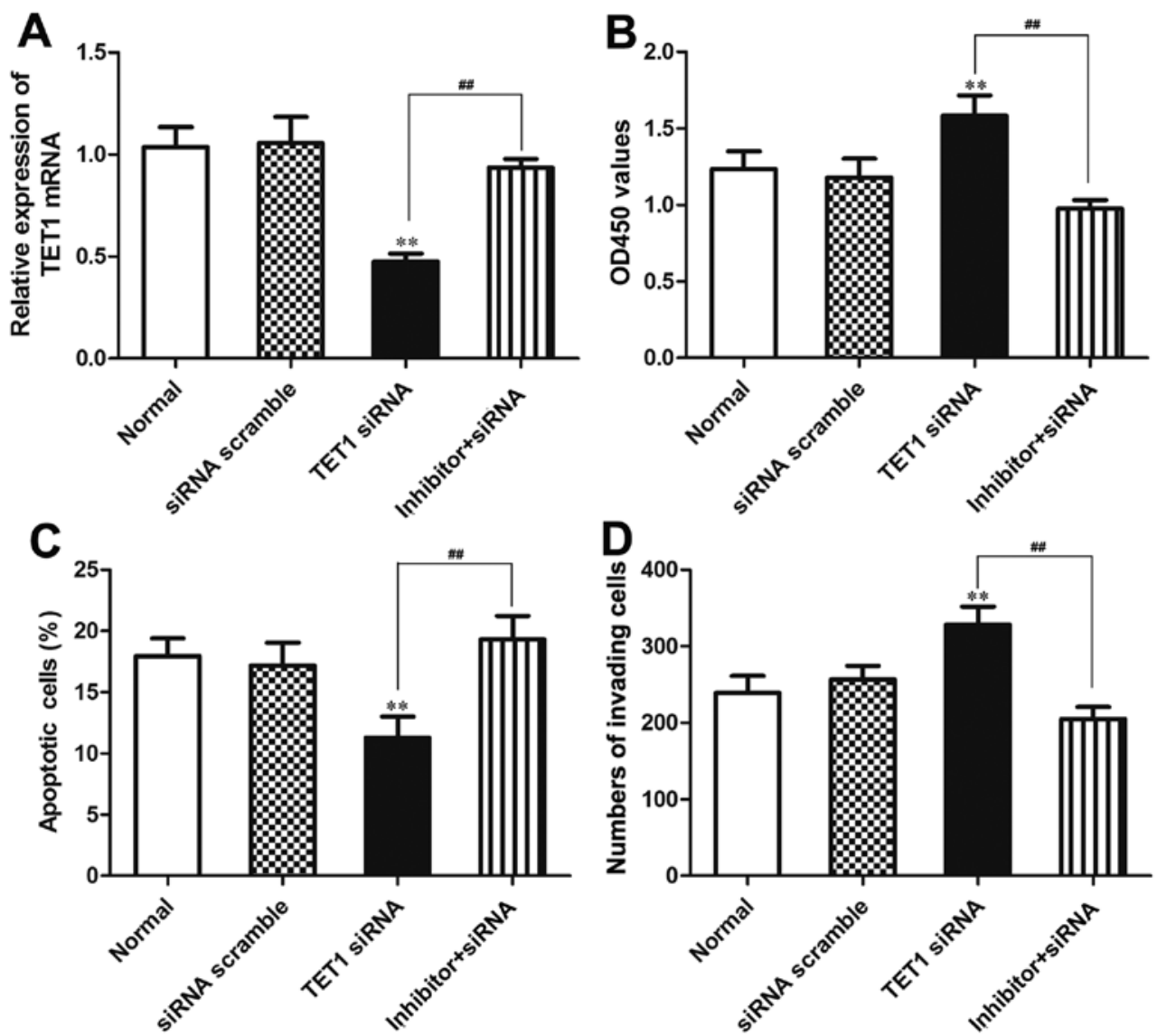

Figure 8. miR-27a-3p affects cellular biological functions in osteosarcoma by targeting TET1. (A) mRNA expression of TET1 in MG-63 cells detected by RT-qPCR; (B) CCK-8 assay was used to detect cell viability. Flow cytometry was used to detect cell apoptosis. Transwell invasion assay was used to detect cell invasion. ${ }^{* *} \mathrm{P}<0.01$ vs. Normal and siRNA scramble groups; ${ }^{\# \#} \mathrm{P}<0.01$.

demonstrated that the number of apoptotic cells in the group co-transfected with miR-27a-3p inhibitor and TET1 siRNA was significantly higher than that in the group transfected with TET1 siRNA $(\mathrm{P}<0.01)$; no significant differences were observed between the normal control group and the siRNA negative control group (Fig. 8C). The results from Transwell 
assay revealed that the number of cells invading through the Transwell chamber in the group co-transfected with the miR-27a-3p inhibitor and TET1 siRNA was significantly lower than that in the group transfected with TET1 siRNA $(\mathrm{P}<0.01)$; no significant differences were found between the normal control group and the siRNA negative control group (Fig. 8D). These data demonstrated that TET1 inhibition attenuated the biological effects induced by miR-27a-3p inhibition in the MG-63 cells, also suggesting that miR-27a-3p affects cellular biological functions by regulating TET1 in osteosarcoma.

\section{Discussion}

In different tumor types, miRNAs play two different roles: As tumor suppressor genes and as oncogenes. Their main mechanisms of action include the loss of loci, amplification, mutations, alterations at the epigenetic level and the abnormal expression of transcription factors $(39,40)$. miR-27a is an evolutionarily conserved family, and has been found to have an abnormal expression in certain types of tumors, such as in breast cancer, gastric cancer and esophageal cancer $(35,41,42)$. However, the role of miR-27a in osteosarcoma has not yet been fully elucidated. Therefore, it is necessary to further examine the role and mechanisms of action of miR-27a in osteosarcoma.

There are a number of studies available on miR-27 and tumors. Zhou et al reported that miR-27a-3p plays the role of a proto-oncogene in gastric cancer via the BAK-SMAC/ DIABLO-XIAP axis (44). miR-27a acts as a proto-oncogene by regulating the expression of MHC class I in colorectal cancer (43). In addition, a number of studies have confirmed that $\mathrm{miR}-27 \mathrm{a}-3 \mathrm{p}$ is related to the sensitivity of cells to chemotherapy and radiotherapy (44). Therefore, in this study, RT-qPCR was used to detect the expression of miR-27a-3p in human osteosarcoma cell lines. The results revealed that the expression of miR-27a-3p was markedly upregulated in the osteosarcoma cell lines compared with the normal osteoblasts, hFOB 1.19 cells, which suggested that miR-27a-3p plays an important role in the development of osteosarcoma.

In order to explore the role of miR-27a-3p in osteosarcoma, the effects of miR-27a-3p on the viability, apoptosis and invasive ability of osteosarcoma cells were determined, and the results revealed that miR-27a-3p inhibition decreased the proliferative ability, contributed to cell apoptosis, and reduced the invasive ability of the osteosarcoma cells. Based on these findings, it can be speculated that miR-27a-3p suppresses the apoptosis of osteosarcoma cells, and promotes the growth and invasion of osteosarcoma cells. Metastasis is the leading cause of mortality in patients with osteosarcoma (45).

The main reason for the distant metastasis of osteosarcoma is the abnormal expression of tumor metastasis-related genes and metastasis suppressor genes $(16,17)$. Increasing evidence has indicated that EMT is associated with the invasive and metastatic behavior of cells during cancer progression $(46,47)$. In the process of EMT in tumors, the expression of epithelial markers (E-cadherin and $\alpha$-catenin) undergoes a downregulation and the expression of mesenchymal markers (vimentin) undergoes an upregulation, which indicates that epitheliumderived tumor cells are losing cell polarity, the connections between cells become loose and intracellular cytoskeletal proteins undergo recombination (48). Therefore, in this study, the expression of EMT-related proteins (E-cadherin, $\alpha$-catenin and vimentin) was detected in the MG-63 cells. The results revealed that miR-27a-3p inhibition decreased the expression of vimentin, and induced the expression of E-cadherin and $\alpha$-catenin, which indicated that the downregulation of miR-27a-3p suppressed EMT in osteosarcoma. On the other hand, it also indicated that miR-27a-3p promoted the expression of vimentin, inhibited by the expression of E-cadherin and $\alpha$-catenin, so as to promote the invasion and migration of osteosarcoma cells.

miRNAs are small (approximately 21 nucleotides in length) non-coding RNAs that play pivotal roles in cellular and developmental processes by regulating gene expression at the post-transcriptional level (49). Our data demonstrated that miR-27a-3p interacted with TET1, and that miR-27a-3p inhibited the expression of TET1 protein in osteosarcoma cells. TET1 inhibits the invasion of cancer cells by inhibiting the methylation of matrix metalloproteinase 2 and 3, and maintaining the expression of tissue inhibitor of matrix metalloproteinases to achieve the inhibition of cancer cell invasion (50). In order to explore whether miR-27a-3p plays a biological role by regulating TET1, miR-27a-3p inhibitor and TET1 siRNA were co-transfected into the MG-63 cells. Our data demonstrated that TET1 inhibition attenuated the biological effects induced by miR-27a-3p inhibition in osteosarcoma cells, also suggesting that miR-27a-3p promoted the malignant phenotypes of osteosarcoma by targeting TET1.

In conclusion, our data demonstrated that miR-27a-3p is upregulated in human osteosarcoma cells. miR-27a-3p inhibition suppressed the viability and invasive ability of the osteosarcoma cells, and promoted cell apoptosis. At the same time, miR-27a-3p inhibition increased the expression of E-cadherin and $\alpha$-catenin, and decreased the expression of vimentin in osteosarcoma cells. Our data also demonstrated that miR-27a-3p interacted with TET1, and TET1 knockdown attenuated the biological effects induced by miR-27a-3p in osteosarcoma cells. on the whole, this study demonstrates that miR-27a-3p promotes the malignant phenotypes of osteosarcoma by targeting TET1; thus, miR-27a-3p may prove to be a potential prognostic marker and therapeutic target for patients with osteosarcoma.

\section{Acknowledgements}

Not applicable.

\section{Funding}

This study was funded by the Natural Science Foundation of Jiangsu Province (grant no. BK20131199), by the fiftyfifth batch of China Postdoctoral Science Foundation (grant no. 2014M551640) and by the Project of Jiangsu provincial Health and Family Planning Commission (grant no. H201524).

\section{Availability of data and materials}

The analyzed datasets generated during the study are available from the corresponding author on reasonable request. 


\section{Authors' contributions}

JZ and JL designed the research and wrote the manuscript. $\mathrm{JL}$ and ML prepared and completed most of the experiments. JL, ML, XL and FL collected the data, and analyzed and interpreted the data. All authors have read and approved the final manuscript.

\section{Ethics approval and consent to participate}

Not applicable.

\section{Consent for publication}

Not applicable.

\section{Competing interests}

The authors declare that they have no competing interests.

\section{References}

1. Bilbao-Aldaiturriaga N, Askaiturrieta Z, Granado-Tajada I, Goričar K, Dolžan V, Garcia-Miguel P, Garcia de Andoin N, Martin-Guerrero I and Garcia-Orad A; For The Slovenian Osteosarcoma Study Group: A systematic review and meta-analysis of MDM2 polymorphisms in osteosarcoma susceptibility. Pediatr Res 80: 472-479, 2016.

2. Anderson PM, Bielack SS, Gorlick RG, Skubitz K, Daw NC, Herzog CE, Monge OR, Lassaletta A, Boldrini E, Pápai Z, et al: A phase II study of clinical activity of SCH 717454 (robatumumab) in patients with relapsed osteosarcoma and Ewing sarcoma. Pediatr Blood Cancer 63: 1761-1770, 2016.

3. Nikitovic D, Kavasi RM, Berdiaki A, Papachristou DJ, Tsiaoussis J, Spandidos DA, Tsatsakis AM and Tzanakakis GN: Parathyroid hormone/parathyroid hormone-related peptide regulate osteosarcoma cell functions: Focus on the extracellular matrix (Review). Oncol Rep 36: 1787-1792, 2016.

4. Liao LQ, Yan HH, Mai JH, Liu WW, Li H, Guo ZM, Zeng ZY and Liu XK: Radiation-induced osteosarcoma of the maxilla and mandible after radiotherapy for nasopharyngeal carcinoma. Chin J Cancer 35: 89, 2016.

5. Bami M, Mavrogenis AF, Angelini A, Milonaki M, Mitsiokapa E, Stamoulis D and Soucacos PN: Bone morphogenetic protein signaling in musculoskeletal cancer. J Cancer Res Clin Oncol 142: 2061-2072, 2016.

6. Angelini A, Mavrogenis AF, Trovarelli G, Ferrari S, Picci P and Ruggieri P: Telangiectatic osteosarcoma: A review of 87 cases. J Cancer Res Clin Oncol 142: 2197-2207, 2016.

7. Wang Z, Li B, Ren Y and Ye Z: T-cell-based immunotherapy for osteosarcoma: Challenges and opportunities. Front Immunol 7: $353,2016$.

8. Osasan S, Zhang M, Shen F, Paul PJ, Persad S and Sergi C: Osteogenic sarcoma: A 21st century review. Anticancer Res 36 : 4391-4398, 2016

9. Nataraj V, Rastogi S, Khan SA, Sharma MC, Agarwala S, Vishnubhatla S and Bakhshi S: Prognosticating metastatic osteosarcoma treated with uniform chemotherapy protocol withou high dose methotrexate and delayed metastasectomy: A single center experience of 102 patients. Clin Transl Oncol 18: 937-944 2016.

10. Hu K, Dai HB and Qiu ZL: mTOR signaling in osteosarcoma: Oncogenesis and therapeutic aspects (Review). Oncol Rep 36: 1219-1225, 2016.

11. Lee JA, Jeon DG, Cho WH, Song WS, Yoon HS, Park HJ, Park BK, Choi HS, Ahn HS, Lee JW, et al: Higher gemcitabine dose was associated with better outcome of osteosarcoma patients receiving gemcitabine-docetaxel chemotherapy. Pediatr Blood Cancer 63: 1552-1556, 2016.

12. Kebudi R, Ozger H, Kızılocak H, Bay SB and Bilgiç B: Osteosarcoma after hematopoietic stem cell transplantation in children and adolescents: Case report and review of the literature. Pediatr Blood Cancer 63: 1664-1666, 2016.
13. Adamopoulos C, Gargalionis AN,Piperi C and Papavassiliou AG: Recent advances in mechanobiology of osteosarcoma. J Cell Biochem 118: 232-236, 2017.

14. Li CJ, Liu XZ, Zhang L, Chen LB, Shi X, Wu SJ and Zhao JN: Advances in bone-targeted drug delivery systems for neoadjuvant chemotherapy for osteosarcoma. Orthop Surg 8: 105-110, 2016.

15. Abarrategi A, Tornin J, Martinez-Cruzado L, Hamilton A, Martinez-Campos E, Rodrigo JP, González MV, Baldini N Garcia-Castro J and Rodriguez R: Osteosarcoma: Cells-oforigin, cancer stem cells, and targeted therapies. Stem Cells Int 2016: 3631764, 2016.

16. Wang SD, Li HY, Li BH, Xie T, Zhu T, Sun LL, Ren HY and Ye ZM: The role of CTLA-4 and PD-1 in anti-tumor immune response and their potential efficacy against osteosarcoma. Int Immunopharmacol 38: 81-89, 2016.

17. Ding L, Congwei L, Bei Q, Tao Y, Ruiguo W, Heze Y, Bo D and Zhihong L: mTOR: An attractive therapeutic target for osteosarcoma? Oncotarget 7: 50805-50813, 2016.

18. Ravindra VM, Eli IM, Schmidt MH and Brockmeyer DL: Primary osseous tumors of the pediatric spinal column: Review of pathology and surgical decision making. Neurosurg Focus 41: E3, 2016.

19. Hurley C, McCarville MB, Shulkin BL, Mao S, Wu J, Navid F, Daw NC, Pappo AS and Bishop MW: Comparison of (18) F-FDG-PET-CT and bone scintigraphy for evaluation of osseous metastases in newly diagnosed and recurrent osteosarcoma. Pediatr Blood Cancer 63: 1381-1386, 2016.

20. Kulcheski FR, Christoff AP and Margis R: Circular RNAs are miRNA sponges and can be used as a new class of biomarker. $\mathrm{J}$ Biotechnol 238: 42-51, 2016.

21. Stope MB, Koensgen D, Weimer J, Paditz M, Burchardt M, Bauerschlag D and Mustea A: The future therapy of endometrial cancer: microRNA's functionality, capability, and putative clinical application. Arch Gynecol Obstet 294: 889-895, 2016.

22. Fang Q, Xu T, Wu C, Zhou S and Sun H: Biotargets in neural regeneration. Biotarget 1: 6, 2017.

23. Wang DD, Chen X, Yu DD, Yang SJ, Shen HY, Sha HH, Zhong SL, Zhao JH and Tang JH: miR-197: A novel biomarker for cancers. Gene 591: 313-319, 2016.

24. Yang C, Zheng SD, Wu HJ and Chen SJ: Regulatory mechanisms of the molecular pathways in fibrosis induced by microRNAs. Chin Med J (Engl) 129: 2365-2372, 2016.

25. Lee $\mathrm{K}$ and Ferguson LR: MicroRNA biomarkers predicting risk, initiation and progression of colorectal cancer. World J Gastroenterol 22: 7389-7401, 2016.

26. Matin F, Jeet V, Clements JA, Yousef GM and Batra J: MicroRNA theranostics in prostate cancer precision medicine. Clin Chem 62: 1318-1333, 2016

27. Rajasekaran S, Pattarayan D, Rajaguru P, Sudhakar Gandhi PS and Thimmulappa RK: MicroRNA regulation of acute lung injury and acute respiratory distress syndrome. J Cell Physiol 231: 2097-2106, 2016.

28. Connelly CM, Moon MH and Schneekloth JS Jr: The emerging role of RNA as a therapeutic target for small molecules. Cell Chem Biol 23: 1077-1090, 2016.

29. Li Y, Li J, Sun X, Chen J, Sun X, Zheng J and Chen R: MicroRNA-27a functions as a tumor suppressor in renal cell carcinoma by targeting epidermal growth factor receptor. Oncol Lett 11: 4217-4223, 2016.

30. Chen S, Sun YY, Zhang ZX, Li YH, Xu ZM and Fu WN: Transcriptional suppression of microRNA-27a contributes to laryngeal cancer differentiation via GSK-3 $\beta$-involved Wnt/ $\beta$ catenin pathway. Oncotarget 8: 14708-14718, 2017.

31. Jiang H, Zhang G, Wu JH and Jiang CP: Diverse roles of miR-29 in cancer (review). Oncol Rep 31: 1509-1516, 2014.

32. Kollinerova S, Vassanelli S and Modriansky M: The role of miR-29 family members in malignant hematopoiesis. Biomed Pap Med Fac Univ Palacky Olomouc Czech Repub 158: 489-501, 2014.

33. Pan W, Wang H, Jianwei R and Ye Z: MicroRNA-27a promotes proliferation, migration and invasion by targeting MAP2K4 in human osteosarcoma cells. Cell Physiol Biochem 33: 402-412, 2014.

34. Zhou L, Liang X, Zhang L, Yang L, Nagao N, Wu H, Liu C, Lin S, Cai G and Liu J: miR-27a-3p functions as an oncogene in gastric cancer by targeting BTG2. Oncotarget 7: 51943-51954, 2016.

35. Hu D and Shilatifard A: Epigenetics of hematopoiesis and hematological malignancies. Genes Dev 30: 2021-2041, 2016. 
36. Forloni M, Gupta R, Nagarajan A, Sun LS, Dong Y, Pirazzoli V, Toki M, Wurtz A, Melnick MA, Kobayashi S, et al: Oncogenic EGFR represses the TET1 DNA demethylase to induce silencing of tumor suppressors in cancer cells. Cell Reports 16: 457-471, 2016.

37. Li L, Li C, Mao H, Du Z, Chan WY, Murray P, Luo B, Chan AT, Mok TS, Chan FK, et al: Epigenetic inactivation of the CpG demethylase TET1 as a DNA methylation feedback loop in human cancers. Sci Rep 6: 26591, 2016.

38. Livak KJ and Schmittgen TD: Analysis of relative gene expression data using real-time quantitative PCR and the 2(-Delta Delta C(T)) method. Methods 25: 402-408, 2001.

39. Zhou K, Liu M and Cao Y: New insight into microRNA functions in cancer: Oncogene-microRNA-tumor suppressor gene network. Front Mol Biosci 4: 46, 2017

40. Kaur S, Lotsari-Salomaa JE, Seppänen-Kaijansinkko R and Peltomäki P: MicroRNA methylation in colorectal cancer. Adv Exp Med Biol 937: 109-122, 2016.

41. Tang W, Zhu J, Su S, Wu W, Liu Q, Su F and Yu F: miR-27 as a prognostic marker for breast cancer progression and patient survival. PLoS One 7: e51702, 2012.

42. Tanaka K, Miyata H, Sugimura K, Fukuda S, Kanemura T, Yamashita K, Miyazaki Y, Takahashi T, Kurokawa Y, Yamasaki M, et al: miR-27 is associated with chemoresistance in esophageal cancer through transformation of normal fibroblasts to cancer-associated fibroblasts. Carcinogenesis 36: 894-903, 2015.

43. Colangelo T, Polcaro G, Ziccardi P, Pucci B, Muccillo L, Galgani M, Fucci A, Milone MR, Budillon A, Santopaolo M, et al: Proteomic screening identifies calreticulin as a miR-27a direct target repressing MHC class I cell surface exposure in colorectal cancer. Cell Death Dis 7: e2120, 2016.
44. Zhou S, Huang Q, Zheng S, Lin K, You J and Zhang X: miR-27a regulates the sensitivity of breast cancer cells to cisplatin treatment via BAK-SMAC/DIABLO-XIAP axis. Tumour Biol 37: 6837-6845, 2016

45. Mirabello L, Koster R, Moriarity BS, Spector LG, Meltzer PS Gary J, Machiela MJ, Pankratz N, Panagiotou OA,Largaespada D, et al: A genome-wide scan identifies variants in NFIB associated with metastasis in patients with osteosarcoma. Cancer Discov 5: 920-931, 2015.

46. Lv YF, Dai H, Yan GN, Meng G, Zhang X and Guo QN: Downregulation of tumor suppressing STF cDNA 3 promotes epithelial-mesenchymal transition and tumor metastasis of osteosarcoma by the Wnt/GSK-3 $\beta / \beta$-catenin/Snail signaling pathway. Cancer Lett 373: 164-173, 2016.

47. Wu Y and Jiang M: The revolution of lung cancer treatment: From vaccines, to immune checkpoint inhibitors, to chimeric antigen receptor T therapy. Biotarget 1: 7, 2017.

48. Sun L and Fang J: Epigenetic regulation of epithelial-mesenchymal transition. Cell Mol Life Sci 73: 4493-4515, 2016.

49. Tufekci KU, Oner MG, Genc S and Genc K: MicroRNAs and multiple sclerosis. Autoimmune Dis 2011: 807426, 2010.

50. Lu HG, Zhan W, Yan L, Qin RY, Yan YP, Yang ZJ, Liu GC, Li GQ, Wang HF, Li XL, et al: TET1 partially mediates HDAC inhibitor-induced suppression of breast cancer invasion. Mol Med Rep 10: 2595-2600, 2014. 